\title{
Définition et épidémiologie des troubles de l'éjaculation
}

\author{
Dominique DELAVIERRE
}

Service d'Urologie-Andrologie, CHR d'Orléans

\section{RÉSUMÉ}

Les troubles de l'éjaculation entrent dans le cadre des dysfonctions sexuelles masculines et regroupent l'éjaculation prématurée et l'anéjaculation.

\section{Ejaculation prématurée}

La définition de l'éjaculation prématurée n'est pas univoque. Trois critères sont importants à prendre en compte : la rapidité de survenue de l'éjaculation, l'absence de contrôle volontaire et la souffrance personnelle et/ou de la partenaire.

L'absence de données physiologiques dans la population générale sur le délai pour éjaculer, les difficultés à définir précisément l'éjaculation prématurée et l'absence de questionnaire ou de méthodes d'évaluation standardisés et validés ne facilitent pas la réalisation des enquêtes et leur comparaison.

Les rares enquêtes effectuées depuis le début des années 1990 montrent toutefois des prévalences élevées puisque la moitié à deux-tiers des hommes présenteraient une éjaculation prématurée au moins occasionnellement et environ $10 \%$ souvent ou tout le temps. L'éjaculation prématurée est apparue plus fréquente que la dysfonction érectile dans plusieurs enquêtes.

Anéjaculation

L'anéjaculation est un terme générique regroupant l'anorgasmie (anéjaculation sans orgasme), dont l'éjaculation tardive ou retardée est une forme mineure, l'éjaculation rétrograde, l'éjaculation sèche et l'éjaculation asthénique.

Il est difficile de dégager des quelques enquêtes publiées des chiffres précis mais la prévalence de l'éjaculation tardive et de l'anorgasmie pourrait certainement être estimée entre 5 et $10 \%$.

\section{Conclusion}

Les troubles de l'éjaculation sont des phénomènes de santé non négligeables avec un impact potentiel sur la sexualité et la fertilité du couple et sa qualité de vie.

Mots clés : éjaculation, éjaculation prématurée, anéjaculation, anorgasmie, éjaculation rétrograde

\section{INTRODUCTION}

Les troubles de l'éjaculation entrent dans le cadre des dysfonctions sexuelles masculines et regroupent l'éjaculation prématurée et l'anéjaculation. Beaucoup moins étudiés que la dysfonction érectile ils sont néanmoins fréquents $[22,24]$.

\section{DEFINITIONS}

\section{Ejaculation prématurée}

La définition de l'éjaculation prématurée (en anglais premature ejaculation) n'est pas univoque et le terme même de prématurée est contesté. De nombreux critères peuvent être pris en compte dans la définition de l'éjaculation prématurée : des critères temporels (en secondes, minutes ou par rapport à la pénétration vaginale), le nombre de mouvements coïtaux, l'insatisfaction de l'homme et de sa partenaire [16].

L'Association Américaine de Psychiatrie (APA) dans le DSM IV a défini l'éjaculation prématurée comme une "éjaculation survenant avant que l'individu ne le souhaite du fait d'une absence réitérée et persistante du contrôle volontaire de l'éjaculation et de l'orgasme pendant la relation sexuelle" [1]. Les experts de la 2ème Consultation internationale sur les dysfonctions sexuelles (Paris 2003) ont insisté sur 3 critères essentiels au diagnostic : rapidité de survenue de l'éjaculation, absence de contrôle volontaire et souffrance personnelle et/ou de la partenaire. Ils ont proposé la définition suivante: "Premature ejaculation is persistent or recurrent ejaculation with minimal stimulation before, on, or shortly after penetration, and before the person wishes it, over which the sufferer has little or no

\section{Correspondance :}

Dr Dominique DELAVIERRE - Service d'Uro-Andrologie, CHR La Source, BP 6709, 45067 ORLEANS Cedex 2 -

Tel 02.38.51.46.26 - Fax 02.38.51.41.59 -

Email delav.uro@wanadoo.fr 
voluntary control which causes the sufferer and/or his partner bother or distress" [16]. Cette définition est proche de celle des recommandations de l'Association Américaine d'Urologie (AUA) publiées en 2004 "Premature ejaculation is ejaculation that occurs sooner than desired, either before or shortly after penetration, causing distress to either one or both partners" [2].

\section{Anéjaculation}

La 1ère Consultation internationale sur la dysfonction érectile (Paris 1999) a proposé les définitions suivantes [14] :

- Delayed ejaculation : undue delay in reaching a climax during sexual activity.

- Anorgasmia : inability to achieve an orgasm during conscious sexual activity, although nocturnal emission may occur.

- Anejaculation : absence of ejaculation during orgasm.

- Retrograde ejaculation : backward passage of semen into the bladder after emission usually due to failure of closure of the bladder neck mechanism, demonstrated by presence of spermatozoa in the urine after orgasm.

En France l'anéjaculation est habituellement définie par l'absence d'éjaculation par le méat urétral malgré une érection normale et une stimulation sexuelle appropriée et prolongée [7]. Cette définition englobe

- l'anorgasmie (anéjaculation sans orgasme), parfois appelée anéjaculation psychogène, dont l'éjaculation tardive ou retardée est une forme mineure ;

- l'éjaculation rétrograde où l'expulsion du sperme s'effectue vers la vessie ;

- l'éjaculation sèche, par défaut d'émission de sperme ;

- l'éjaculation asthénique ou baveuse, liée à une altération de l'expulsion du sperme, qui se rapproche de l'anéjaculation en raison de facteurs de risque communs.

\section{EPIDÉMIOLOGIE}

Depuis le début des années 1990 nous avons relevé dans la littérature mondiale plusieurs enquêtes épidémiologiques essentiellement réalisées dans la population générale.

\section{En France}

L'enquête Spira/Bejin (ACSF), réalisée au téléphone de septembre 1991 à février 1992 en raison de l'épidémie de $\mathrm{VIH}$, étudiait les comportements sexuels des français et comportait 7 questions sur les dysfonctions sexuelles. 1308 hommes de 18 à 69 ans et ayant déjà eu au moins un rapport sexuel ont été interrogés. Le Tableau 1 détaille les questions concernant l'éjaculation. L'analyse des réponses, réalisée par Béjin, a fait apparaître que $65 \%$ présentaient "au moins occasionnellement" une éjaculation prématurée (5\% avant la pénétration vaginale, $24 \%$ pendant ou après et $36 \%$ avant, pendant ou après), $73 \%$ une éjaculation retardée ou absente, et $34 \%$ une anorgasmie. En ne prenant en compte que les réponses "souvent" les prévalences étaient de $11 \%$ pour l'éjaculation prématurée ( $1 \%$ avant la pénétration vaginale, $6 \%$ pendant ou après et $4 \%$ avant, pendant ou après), $6 \%$ pour l'éjaculation retardée ou absente, et $7 \%$ pour l'anorgasmie $[5,25]$.

\section{Aux USA}

Dans l'enquête de Laumann (NHSLS), 1410 hommes de 18 à 59 ans, représentatifs de la population générale des USA mais ne vivant pas dans des casernes, dortoirs ou prisons, ayant eu au moins 1 partenaire dans les 12 mois précédents, ont répondu, en 1992, à 7 questions sur leur fonction sexuelle dans les 12 mois précédents. 30,7\% (parmi 1243 réponses exploitables - taux de réponses $88,2 \%$ ) présentaient un orgasme trop rapide et $7,8 \%$ (parmi 1246 réponses exploitables - taux de réponses $88,4 \%$ ) une anorgasmie [15].

\section{En Suède}

Dans l'enquête de Fugl-Meyer et al., 1475 hommes de 18 à 74 ans ont répondu en 1996 à un questionnaire sur leur fonction sexuelle pendant l'année passée au cours d'une interview face à face. Le Tableau 2 détaille les questions sur l'éjaculation. $53 \%$ (parmi 1281 réponses exploitables - taux de réponses $86,8 \%$ ) présentaient une éjaculation prématurée "au moins occasionnellement" dont $9 \%$ "souvent, presque tout le temps ou tout le temps" et $41 \%$ (parmi 1265 réponses exploitables - taux de réponses $85,8 \%$ ) une éjaculation retardée "au moins occasionnellement" dont $2 \%$ "souvent, presque tout le temps ou tout le temps" [12].

\section{En Angleterre}

a) Dans l'enquête de Dunn et al., 789 hommes ont répondu en 1996 à un questionnaire sur leur fonction sexuelle envoyé par la poste à un échantillon de 2000 hommes de 18 à 75 ans randomisé dans 4 cabinets de médecine générale représentatifs d'Angleterre (taux de réponse de $39 \%$ ). Le Tableau 3 précise la question sur l'éjaculation. $31 \%$ signalaient une éjaculation prématurée au cours de leur vie et $14 \%$ dans les 3 mois précédents sans précision de fréquence [10].

Egalement en Angleterre, 2 enquêtes publiées en 1997 ont concerné de petits échantillons.

b) L'enquête de Read et al. a été effectuée dans un cabinet de médecine générale du nord de Londres chez 72 hommes de 18 ans ou plus. Cette enquête a utilisé le questionnaire GRISS (Golombok-Rust Inventory of Sexual Satisfaction) dont les questions sur l'éjaculation sont détaillées dans le Tableau 4 [23]. Vingt deux hommes (31\%) présentaient une éjaculation prématurée [21].

c)L'enquête de Goldmeier et al. a été effectuée dans un service de médecine uro-génitale d'un hôpital du centre de Londres chez 106 hommes de 16 à 78 ans consultant pour la 1ère fois dans ce service. Cette enquête a aussi utilisé le questionnaire GRISS. Vingt trois hommes (22\%) présentaient une éjaculation prématurée [13].

\section{Aux Pays-bas}

Dans l'enquête de Blanker et al., un questionnaire sur leur 
Tableau 1 : Questions sur l'éjaculation de l'enquête Spira/Béjin [5, 25].

Avez vous déjà présenté les signes suivants ?

Vous éjaculez trop rapidement avant même de pénétrer votre partenaire.

Vous éjaculez trop rapidement au moment de la pénétration ou peu après elle.

Vous mettez trop longtemps à éjaculer une fois que vous avez pénétré votre partenaire.

Vous n'arrivez pas à éjaculer du tout.

Vous n'avez aucun orgasme.

Tableau 2 : Questions sur l'éjaculation de l'enquête Fugl-Meyer et al. [12].

It happens that the man has ejaculation very shortly after intromission. Has this occurred in your sexual life during the last 12 months?

It happens that the man has difficulties in achieving ejaculation. Has this occurred in your sexual life during the last 12 months ?

Tableau 3 : Question sur l'éjaculation de l'enquête de Dunn et al. [10].

Have you had difficulty with ejaculating prematurely in the past three months / ever ?

Tableau 4 : Questions sur l'éjaculation du questionnaire GRISS [23].

Can you avoid ejaculation too quickly during intercourse?

Do you ejaculate without wanting to almost as soon as your penis enters your partner's vagina? 
fonction sexuelle a été proposé d'août 1995 à janvier 1998 à 3924 hommes de 50 à 78 ans, n'ayant pas été opérés d'une prostatectomie radicale, dans une ville proche de Rotterdam (1605 réponses exploitables : 40,9\%). Cette enquête n'a pas relevé les éjaculations prématurées ni les anéjaculations telles que définies précédemment mais a signalé que $13,4 \%$ des hommes interrogés présentaient une réduction de volume de leur éjaculât voire une absence totale [6].

\section{En Australie}

Dans l'enquête de Pinnock et al. publiée en 1999, 745 hommes d'Australie Méridionale de 40 ans ou plus ont reçu un questionnaire par la poste évaluant 7 domaines de la fonction sexuelle dans les trois mois précédents. Le taux de réponses exploitables a été de $49,8 \%$ (371 hommes - âge moyen 57,5 ans). $18,2 \%$ signalaient un orgasme "atténué ou absent" [20].

\section{En Allemagne}

Dans l'enquête d'Aschka et al. publiée en 2001, 307 hommes de 18 ans ou plus ont répondu à un questionnaire sur leur fonction sexuelle au cabinet de vingt médecins de famille (taux de réponse $84 \%$ ). $60 \%$ présentaient une éjaculation prématurée "au moins occasionnellement" dont $11 \%$ "souvent ou toujours" [3].

En 2001 et 2002 l'enquête GSSAB (Global Study of Sexual Attitudes and Behaviors), dirigée par Nicolosi, a été effectuée dans 29 pays de diverses régions du monde chez 145 380 hommes ou femmes de 40 à 80 ans avec pour objectifs de préciser leurs comportements sexuels et la prévalence des dysfonctions sexuelles. Selon les pays les interviews ont été réalisées lors d'un entretien direct, par téléphone ou par voie postale et le taux de réponse a été de 19\%. 13618 hommes ont complété le questionnaire. 14\% présentaient "parfois" ou "souvent" une éjaculation prématurée (appelée early ejaculation dans cette enquête) et $7 \%$ "parfois" ou "souvent" une anorgasmie. Les prévalences de ces troubles de l'éjaculation étaient les plus fréquentes en Asie du Sud-Est (25\% pour l'éjaculation prématurée et $15 \%$ pour l'anorgasmie) et les moins fréquentes au MoyenOrient pour l'éjaculation prématurée $(8 \%)$ et en Europe du Nord pour l'anorgasmie (5\%) [19].

\section{Autres}

Trois enquêtes récentes ont évalué la prévalence de l'éjaculation prématurée dans certains groupes de patients :

- En Arabie Saoudite El-Sakka (à Makkah), a réalisé de juillet 2001 à décembre 2002 une enquête chez 676 hommes de 27 à 75 ans atteints de diabète type 2. La prévalence de l'éjaculation prématurée a été évaluée à $40,2 \%$. Seules les éjaculations prématurées secondaires apparues depuis la découverte du diabète ont été prises en compte et non les éjaculations primaires. L'éjaculation prématurée était 2,7 fois plus fréquente chez les patients souffrant de diabète depuis plus de 10 ans que chez les patients souffrant de diabète depuis moins de 5 ans et 9,6 fois plus fréquente chez les patients dont le contrôle métabolique était mauvais $(\mathrm{HbA} 1 \mathrm{c}>7 \%)$ que chez les autres [11].

- En Turquie l'enquête d'Aslan et al. (à Izmir), publiée en 2003 à propos de 98 hommes âgés de 30 à 69 ans hémodialysés sans dysfonction érectile ni autres troubles sexuels, a chiffré la prévalence de l'éjaculation prématurée à $31,6 \%$ [4].

- En Chine dans l'enquête de Liang et al. (à Heifei), publiée en 2004 à propos de 1768 hommes âgés de 20 à 59 ans atteints de prostatite chronique définie selon les critères du groupe d'experts du National Institutes of Health sur les prostatites chroniques [18], cette prévalence a été chiffrée à $33,9 \%$ [17].

\section{DISCUSSION}

En 1990, dans un article de revue, Spector et Carey évaluaient la prévalence de l'éjaculation prématurée à $36-38 \%$ contre $4-9 \%$ pour la dysfonction érectile et $4-10 \%$ pour l'inhibition de l'orgasme [24]. Plus récemment, en 2000, également dans un article de revue, Rosen avançait des prévalences comparables : $30 \%$ pour l'éjaculation prématurée, $10-20 \%$ pour la dysfonction érectile et $15 \%$ pour l'insuffisance de désir sexuel [22]

Contrairement aux enquêtes concernant la dysfonction érectile, les enquêtes épidémiologiques publiées depuis le début des années 1990 dans le domaine des troubles de l'éjaculation sont rares mais confirment des prévalences non négligeables (Tableau 5). Ces enquêtes montrent notamment que la moitié à deux-tiers des hommes présenteraient une éjaculation prématurée "au moins occasionnellement" et l'éjaculation prématurée est apparue plus fréquente que la dysfonction érectile dans les enquêtes d'Aschka, Fugl-Meyer, Goldmeier, Laumann, Nicolosi, Read, et Spira/Béjin $[3,5,12,13,15,19,21,25]$. La prévalence de l'éjaculation prématurée survenant "souvent ou tout le temps" pourrait être estimée autour de 10\%.

L'absence de données physiologiques dans la population générale sur le délai pour éjaculer, les difficultés à définir précisément l'éjaculation prématurée et l'absence de questionnaire ou de méthodes d'évaluation standardisés et validés ne facilitent pas la réalisation des enquêtes et leur comparaison. L'impact réel d'une éjaculation survenant rapidement ou prématurément sur la qualité des relations sexuelles et la qualité de vie du couple n'a pas été évalué. Comme l'indique Marie-Hélène Colson l'éjaculation prématurée n'est pas une maladie mais un "symptôme porté par l'homme mais généré par un couple où il n'est pas possible de trouver un langage commun d'accession à un plaisir partagé" [8]. C'est pourquoi il est important de considérer la souffrance de l'homme et/ou de sa partenaire avant de parler d'éjaculation prématurée significative.

L'anéjaculation est un terme générique regroupant diverses situations. La première étape de l'évaluation d'une anéjaculation est de la classifier et de préciser la présence ou non d'un orgasme. Toutefois l'appellation d'anéjaculation psychogène volontiers employée pour désigner l'anorgas- 
Tableau 5 : Prévalence de l'éjaculation prématurée.

\begin{tabular}{ccccccc}
\hline Auteurs & Pays & Année & $\begin{array}{c}\text { Nombre } \\
\text { d'hommes }\end{array}$ & $\begin{array}{c}\text { Age } \\
\text { (ans) }\end{array}$ & Définition & Prévalence \\
\hline $\begin{array}{c}\text { Spira/Béjin } \\
{[5,25]}\end{array}$ & France & $1991-92$ & 1308 & 18 à 69 & $\begin{array}{c}\text { Ejaculation prématurée } \\
\text { "souvent" }\end{array}$ & $11 \%$ \\
$\begin{array}{c}\text { Laumann et al. } \\
{[15]}\end{array}$ & USA & 1992 & 1243 & 18 à 59 & $\begin{array}{c}\text { Orgasme trop rapide } \\
\text { dans les } 12 \text { derniers mois }\end{array}$ & $30,7 \%$ \\
$\begin{array}{c}\text { Fugl-Meyer et al. } \\
{[12]}\end{array}$ & Suède & 1996 & 1281 & 18 à 74 & $\begin{array}{c}\text { "souvent, presque tout le } \\
\text { temps ou tout le temps" } \\
\text { dans les 12 derniers mois }\end{array}$ & $9 \%$ \\
$\begin{array}{c}\text { Dunn et al. } \\
{[10]}\end{array}$ & Angleterre & 1996 & 789 & 18 à 75 & $\begin{array}{c}\text { Ejaculation prématurée } \\
\text { dans les 3 derniers mois }\end{array}$ & $14 \%$ \\
$\begin{array}{c}\text { Aschka et al. } \\
{[3]}\end{array}$ & Allemagne & 2001 & 307 & 18 & $\begin{array}{c}18 \\
\text { ou plus } \\
\text { Ejaculation prématurée }\end{array}$ & "souvent ou tout le temps" \\
$\begin{array}{c}\text { Nicolosi et al. } \\
{[19]}\end{array}$ & $\begin{array}{c}\text { Enquête } \\
\text { mondiale }\end{array}$ & $2001-02$ & 13618 & 40 à 80 & $\begin{array}{c}\text { Ejaculation prématurée } \\
\text { "parfois" ou "souvent" }\end{array}$ & $14 \%$ \\
\hline
\end{tabular}

mie est impropre et devrait être réservée à l'anorgasmie primaire [9]. Il est difficile de dégager des quelques enquêtes publiées des chiffres précis mais la prévalence de l'éjaculation tardive et de l'anorgasmie pourrait certainement être estimée entre 5 et $10 \%$.

\section{CONCLUSION}

Les troubles de l'éjaculation sont en conséquence des phénomènes de santé non négligeables avec un impact potentiel sur la sexualité et la fertilité du couple et sa qualité de vie.

\section{RÉFÉRENCES}

1. American Psychiatrical Association, Guelfi J.D. DSM IV text revision. Manuel diagnostique et statistique des troubles mentaux. Paris, Masson, 2003.

2. American Urological Association. Guideline on the pharmacologic treatment of premature association. Baltimore, American Urological Association Inc ${ }^{R}, 2004$.

3. ASCHKA C., HIMMEL W., ITTNER E., KOCHEN M.M. : Sexual problems of male patients in family practice. J. Fam. Pract., 2001, $50: 773-778$.

4. ASLAN G., ARSLAN D., CAVDAR C., SIFIL A., ESEN A.A., CAMSARI T. : Analysis of premature ejaculation in hemodialysis patients using the International Index of Erectile Function. Urol. Int., 2003, $70: 59-61$.

5. BEJIN A. : Epidémiologie de l'éjaculation prématurée et de son cumul avec la dysfonction érectile. Andrologie, 1999, $9: 211$ 225.
6. BLANKER M.H., BOSCH J.L., GROENEVELD F.P. et at. : Erectile and ejaculatory dysfunction in a community-based sample of men 50 to 78 years old : prevalence, concern, and relation to sexual activity. Urology, 2001, $57: 763-768$.

7. BUVAT J., LEMAIRE A., MARCOLIN G., BUVAT-HERBAUT M.: Ejaculation retardée et anéjaculation sans orgasme. A propos des résultats des explorations et du traitement dans 60 cas. Acta Urol. Belg., 1989, 57 : 195-206.

8. COLSON M.H. : Trop vite, moi ? In : Réaliser sa sexualité. Paris, Pocket évolution, 2003, 85-102.

9. DELAVIERRE D., HAPI M.H., NSABIMBONA B. : Diagnostic d'une anéjaculation. A propos de 72 patients. Andrologie, 2004 , $14: 164-170$.

10. DUNN K.M., CROFT P.R., HACKETT G.I. : Sexual problems : a study of the prevalence and need for health care in the general population. Fam. Pract., 1998, $15:$ 519-524.

11. El-SAKKAA. : Premature ejaculation in non-insulin-dependent diabetic patients. Int. J. Androl., 2003, 26 : 329-334.

12. FUGL-MERYER A.R., SJOGREN FUGL-MERYER K. : Sexual disabilities, problems and satisfaction in 18-74 year old Swedes. Scand. J. Sexol., 1999, 2 : 79-105.

13 GOLDMEIER D., KEANE F.E.A., CARTER P., HESSMAN A., HARRIS J.R.W., RENTON A. : Prevalence of sexual dysfunction in heterosexual patients attending a central London genitourinary medicine clinic. Int. J. STD AIDS, 1997, $8: 303-306$.

14. HENDRY W.F., ALTHOF S.E., BENSON G.F. et al. : Male orgasmic and ejaculatory disorders. In : Jardin A., Wagner G., Khoury S. et al. eds. Erectile dysfunction. Paris, Health publication Ltd, 2000, 477-506.

15. LAUMANN E.O., PAIK A., ROSEN R.C. : Sexual dysfunction in the United States : prevalence and predictors. J. Am. Med. Ass., 1999, 281 : 537-544. 
16. LEWIS R.W., FUGL-MERYER K.S., BOSCH R. et al. : Definitions, classification and epidemiology of sexual dysfunction. In : Lue TF, Basson R., Rosen R. et al. eds. Paris, Health publication Ltd, 2004, 39-72.

17. LIANG C.Z., ZHANG X.J., HAO Z.Y., SHI H.Q., WANG K.X. : Prevalence of sexual dysfunction in Chinese men with chronic prostatitis. Br. J. Urol. Int., 2004, 93 : 568-570.

18. LITWIN M.S., MCNAUGHTON-COLLINS M., FOWLER F.J. Jr et al. : The National Institutes of Health chronic prostatitis symptom index : development and validation of a new outcome measure. Chronic Prostatitis Collaborative Research Network. J. Urol., 1999, $162:$ 369-375.

19. NICOLOSI A., LAUMANN E.O., GLASSER D.B., MOREIRA E.D., PAIK A., GINGELL C. : Sexual behavior and sexual dysfunctions after age 40 : the Global Study of Sexual Attitudes and Behaviors. Urology, 2004, 64 : 991-997.

20. PINNOCK C.B., STAPLETON A.M., MARSHALL V.R. : Erectile dysfunction in the community: a prevalence study. Med. J. Aust., 1999, $171: 353-357$.

21. READ S., KING M., WATSON J. : Sexual dysfunction in primary medical care : prevalence, characteristics and detection by the general practitioner. J. Public Health Med., 1997, $19: 387-$ 391.

22. ROSEN RC. : Prevalence and risk factors of sexual dysfunction in men and women. Curr. Psychiatry Rep., 2000, 2 : 189195.

23. RUST J., GOLOMBOK S. : The Golombok Rust inventory of sexual satisfaction. Br. J. Clin. Psychol., 1985, 24 : 63-64.

24. SPECTOR I.P., CAREY M.P. : Incidence and prevalence of the sexual dysfunctions : a critical review of the empirical literature. Arch. Sex. Behav., 1990, $19: 389-408$.

25. SPIRA A., BAJOS N. et le groupe ACSF : Les comportements sexuels en France. Paris, La documentation française, 1993.

Manuscrit reçu : mai 2005 ; accepté juin 2005.

\section{ABSTRACT}

Definition and epidemiology of ejaculatory dysfunction

Dominique DELAVIERRE

Ejaculatory dysfunction is a male sexual disorder and comprises premature ejaculation, delayed ejaculation, anorgasmia, anejaculation and retrograde ejaculation. The definition of premature ejaculation is based on three essential criteria: brief ejaculatory latency, loss of control and psychological distress for the patient and/or his partner.

Comparison of studies on premature ejaculation is difficult due to the absence of physiological data in the general population on ejaculatory latency, the absence of a precise definition of premature ejaculation and the absence of a questionnaire or standardized and validated methods of evaluation. However, the rare studies performed since 1990 show high prevalences: about $10 \%$ of men often or always experience premature ejaculation.

The prevalence of delayed ejaculation and anorgasmia is estimated to be between 5 and $10 \%$.

Ejaculatory dysfunctions are therefore significant health problems with consequences on sexuality, fertility and quality of life.

Key words: ejaculation, premature ejaculation, delayed ejaculation, anorgasmia, anejaculation, retrograde ejaculation 